\title{
Open-end tube dynamic flow model with an oscillatory extortion
}

\author{
Tytus Tulwin ${ }^{1, *}$, Monika Klimek ${ }^{2}$, and Piotr Piech ${ }^{2}$ \\ ${ }^{1}$ Lublin University of Technology, Mechanical Engineering Faculty, ul. Nadbystrzycka 36., 20-618 Lublin, Poland \\ ${ }^{2}$ Medical University of Lublin, Department of Human Anatomy, al. Racławickie 1, 20-059 Lublin, Poland
}

\begin{abstract}
This paper presents a derivation of dynamic $2 \mathrm{~d}$ mathematical model for open end tube with oscillatory extortion in the region of the closed end. The aim the research is to investigate possible uses of the increased pressure in the enclosed tube chamber, especially for energy efficient lift generation. The mathematical model allows to test and predict how flow modifications impact the resultant lifting force. A derivation of the proposed mathematical model is shown. The mathematical model is then compared to the computational fluid dynamics discrete model. The results prove the accuracy of the mathematical physical model.
\end{abstract}

\section{Introduction}

In fluid dynamics it is difficult to model complex scenarios, especially when turbulence and viscosity are involved. Omitting those terms can lead to a simplified mathematical model that can represent real world scenarios with good accuracy. This paper presents a derivation of dynamic $2 \mathrm{~d}$ mathematical model for open end tube with oscillatory extortion in the region of the closed end. The other end of the tube is open. An open-air tube is often used in musical instruments as it can intensify the sound pressure level at resonant frequencies. This phenomenon known as acoustic resonance. This paper presents the results of the resonant pressure buildup experiment. The aim of the research is to investigate possible uses of the increased pressure in the enclosed tube chamber. Especially for energy efficient lift generation. The mathematical model allows to test and predict how flow modifications impact the resultant lifting force. There are studies investigating dynamic lift force acting on tube arrays [1][2][3][4][5].

\section{Materials and methods}

The experiment is based on the square profile $0.5 \mathrm{~m}$ long tube, with extra $0.1 \mathrm{~m}$ pressure chamber with two oscillatory membranes. The tube width is $0.1 \mathrm{~m}$. The investigation consists of three approaches: theoretical, CFD simulation and experimental. The frequency is set to $400 \mathrm{~Hz}$ with oscillatory membrane displacement close to $1 \mathrm{~cm}$. The Computational Fluid Dynamics model is based on the same geometry, with hemispherical open field conditions. The theoretical model is simplified to the onedimensional problem, basing on Euler governing energy equation. The experimental setup is visible in figure 1 . The dimensions and parameters for theoretical model are presented in figure 2 .

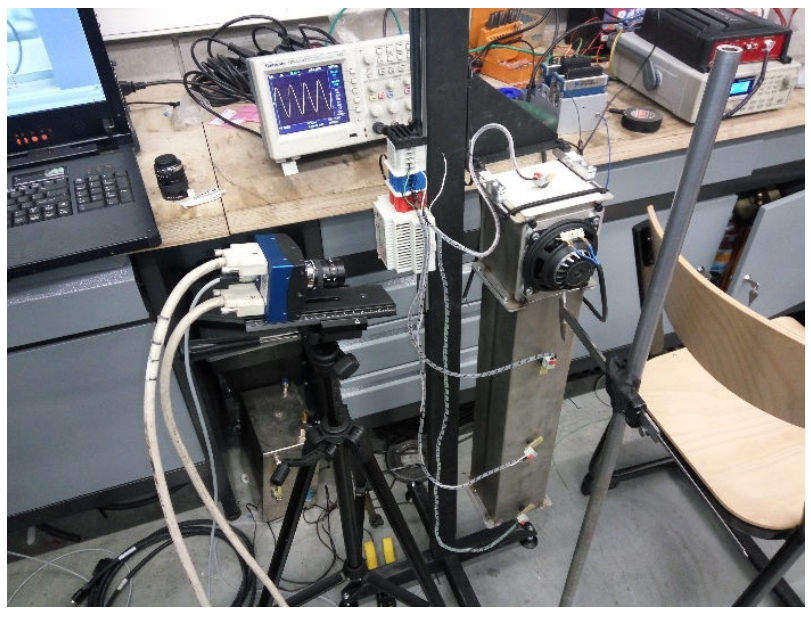

Fig. 1. The open resonance tube experimental setup.

The boundary condition of pressure $\mathrm{P} 2$ is set to $0 \mathrm{~Pa}$ relative to the atmospheric pressure, because it is a standing wave node. $\mathrm{P} 0$ is the maximum pressure extorted by the oscillatory membranes.

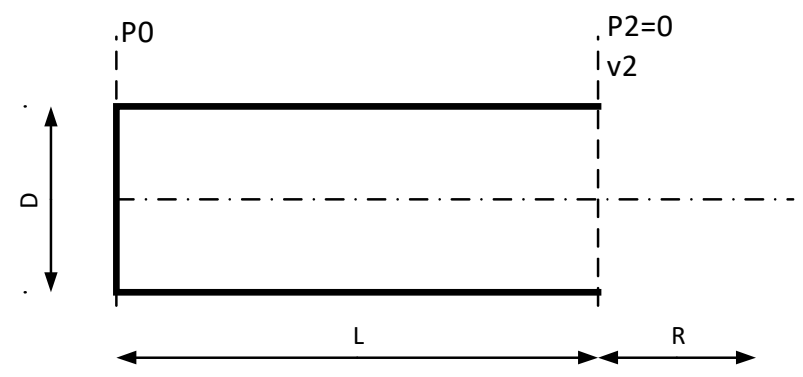

Fig. 2. The opn-tube dimensions and parameters.

A well-known Euler equation is used and simplified to the one-dimensional form:

\footnotetext{
${ }^{*}$ Corresponding author: t.tulwin@pollub.pl
} 


$$
\frac{\partial \bar{v}}{\partial t}+(\bar{v} \operatorname{grad} \bar{v})=g-\operatorname{grad} p
$$

which can be written in differential form:

$$
\frac{\partial v}{\partial t}+v \frac{\partial v}{\partial s}=\frac{1}{\rho} \frac{\partial p}{\partial s}
$$

Dividing both sides of the equation 1 by $\partial s$ leads to equation 3 . The first term corresponds to the transient inertial effect.

$$
\frac{\partial v}{\partial t} d s+v d v=\frac{1}{\rho} d p
$$

At this point every term in the equation 3 can be integrated with known integration limits as shown in equation 4 :

$$
\int_{0}^{L+R} \frac{\partial v}{\partial t} d s+\int_{v_{0}}^{v_{2}} v d v=\int_{p_{0}}^{p_{2}} \frac{1}{\rho} d p
$$

The dimensional limits correspond to the tube location along its axis as shown in figure 3. The distance $\mathrm{R}$ corresponds to the area of effect in open field region. For simplicity it was assumed that the velocity profile decays in linear way with distance. For higher accuracy this profile could be represented by inverse distance equation.

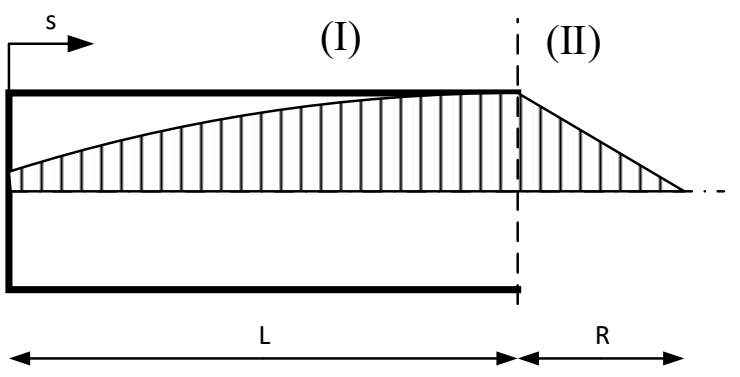

Fig. 3. A predicted velocity profile along the tube's axis required for acceleration integration in expanded bernouli equation.

This leads to the modified unsteady Bernoulli equation 5 $[6][7][8]$.

$$
\int_{0}^{L+R} \frac{\partial v}{\partial t} d s+\frac{1}{2}\left(v_{2}^{2}-V_{0}^{2}\right)=\left(\frac{k}{k-1}\right) \frac{p_{2}-p_{0}}{\rho_{0}}
$$

The transient term needs to be integrated along the axial distance with limits covering whole region of its effect. The air outside the pipe has still significant influence on forces present. It is crucial to predict the shape of the acceleration profile in respect to the axial distance. Inside the pipe the shape is sinusoidal as the generated extortion signal is also sinusoidal. Outside the pipe air enters hemispherical open field region. The profile of acceleration still has a sinusoidal nature but decays quickly inversely proportional to distance (Fig. $4,5)$. It can be assumed that outside the tube air pressure and velocity are inversely proportional to the axis distance with sinusoidal term. For a frequency of $400 \mathrm{~Hz}$ the sinusoidal term can be omitted for simplification.

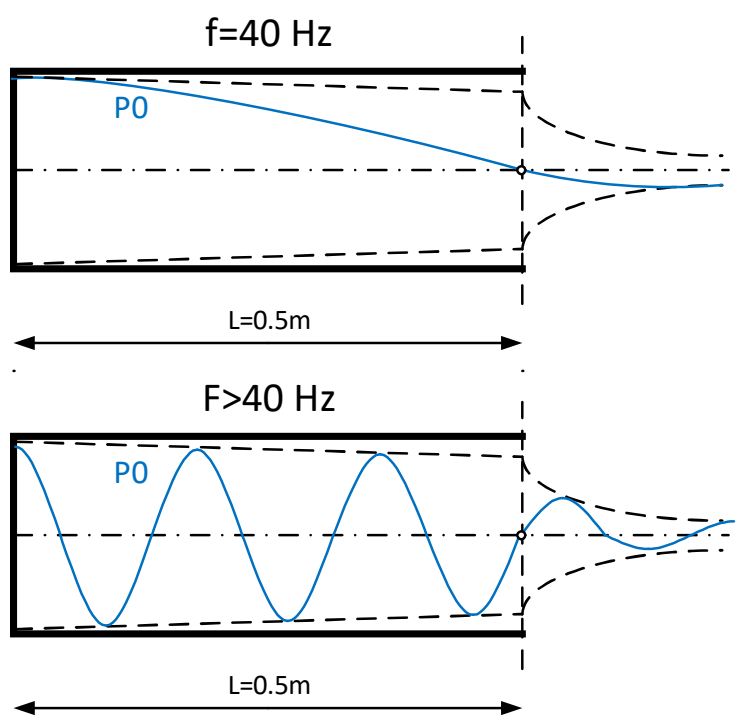

Fig. 4. Pressure distribution along the tube's axis for two different frequency levels.

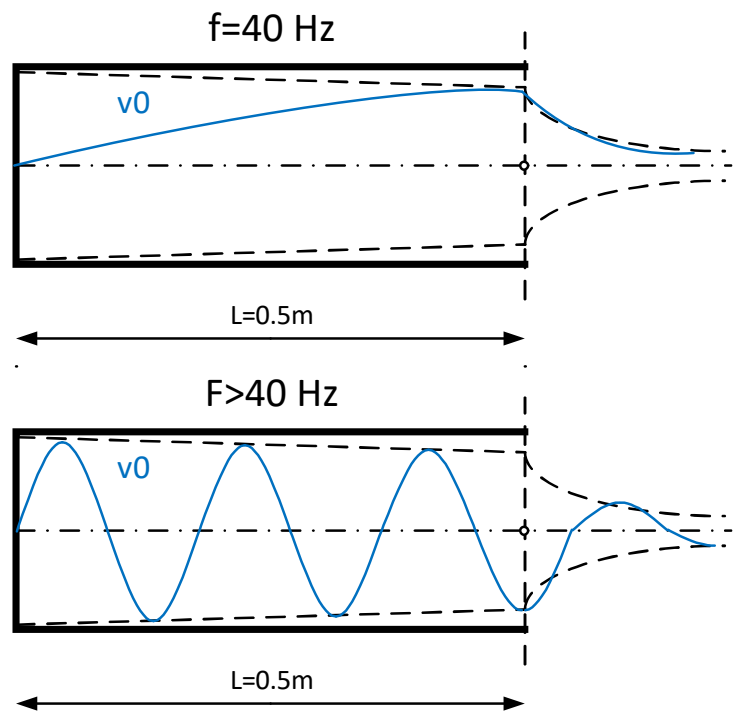

Fig. 5. Velocity distribution along the tube's axis for two different frequency levels.

In order to integrate the transient term of equation 5 it is split to two regions: I amd II. Region I represent the control volume inside the tube and region II represent the control volume outside the tube.

$$
\int_{0}^{L+R} \frac{\partial v}{\partial t} d s=\int_{0}^{L} \frac{\partial v_{(I)}}{\partial t} d s+\int_{0}^{L+R} \frac{\partial v_{(I I)}}{\partial t} d s
$$

Inside region I the velocity follows sinusoidal path, that can be written as:

$$
v_{2(I)}(s)=-\cos \left(\frac{s-L}{c} 2 \pi f\right) v_{2}
$$

Inside region II the velocity profile is considered as linear decaying to 0 at a distance value of $\mathrm{R}$ : 


$$
v_{2(I I)}(s)=\frac{(R-(s-L))}{R} v_{2}
$$

Combining equation 7 and 8 gives:

$$
\begin{aligned}
\int_{0}^{L+R} \frac{\partial v}{\partial t} d s=\int_{0}^{L+R} & \frac{v_{2}-v_{2(t-1)}}{\Delta t} d s \\
& =\int_{0}^{L} \frac{v_{2(I)}-v_{2(I)(t-1)}}{\Delta t} d s \\
& +\int_{0}^{L+R} \frac{v_{2(I I)}-v_{2(I I)(t-1)}}{\Delta t} d s
\end{aligned}
$$

Equation 9 is then integrated:

$$
\begin{gathered}
\int_{0}^{L+R} \frac{\partial v}{\partial t} d s=\frac{1}{\Delta t}\left(v_{2}-v_{2(t-1)}\right)\left[\frac{c}{2 \pi f}\left(-1+\sin \left(\frac{L}{c} 2 \pi f\right)\right)\right. \\
\left.+\frac{1}{2} R+\frac{L^{2}}{2 R}\right]
\end{gathered}
$$

Equation 10 can now be inserted to equation 5, giving the final governing equation of the mathematical model:

$$
\begin{aligned}
\frac{1}{\Delta t}\left(v_{2}-v_{2(t-1)}\right) & {\left[\frac{c}{2 \pi f}\left(-1+\sin \left(\frac{L}{c} 2 \pi f\right)\right)+\frac{1}{2} R+\frac{L^{2}}{2 R}\right] } \\
& +V_{2}^{2} \frac{1}{2}\left(1+\lambda \frac{L}{D}+\xi\right)+\left(\frac{k}{k-1}\right) \frac{p_{2}-p_{0}}{\rho} \\
& -\frac{1}{\Delta t}\left[\frac{c}{2 \pi f}\left(1+\sin \left(\frac{L}{c} \frac{c}{2 \pi f}\right)\right)+\frac{1}{2} R\right. \\
+ & \left.\frac{L^{2}}{2 R}\right] v_{2(t-1)}=0
\end{aligned}
$$

The viscosity loss is added in form of Darcy-Weisbach loss coefficients. Useful one-dimension governing equations can be found in paper [9] In this paper the author presented a different pressure wave equation [10].

\section{Results and discussion}

The results show that 2D model follows closely the CFD simulated pressure and velocity distribution along the tube axis (Fig 6).

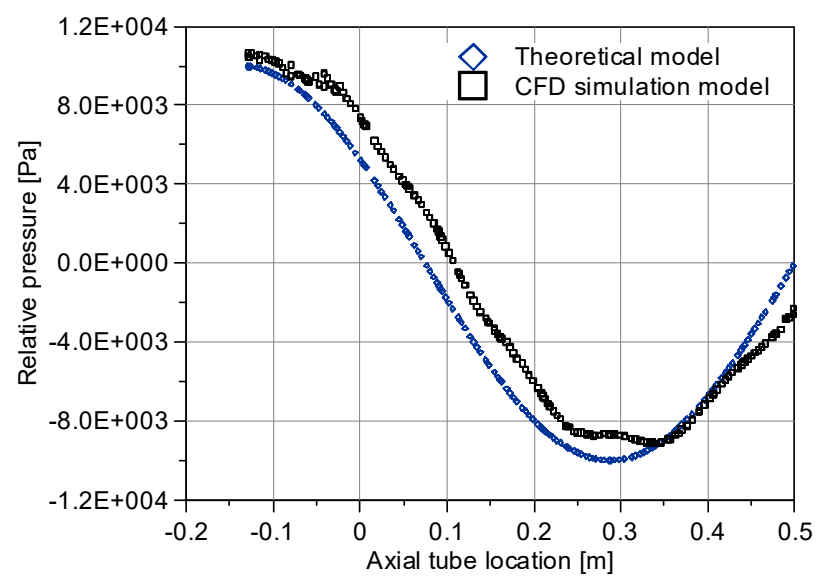

Fig. 6. Relative pressure distribution along the tube axis. The open end is on the right side.
Highest pressure is observed near the oscillation generator membranes on the left side of the tube. On the second antinode of the standing wave the amplitude drops slightly because of the viscous wall losses. A last pressure node of the confined tube is located at the end of the tube. A good representation of standing waves was found in paper [11]. CFD and theoretical models seem to overlap accurately with slight shift along $\mathrm{x}$ axis. This shift may be caused by differences in model geometry. The mathematical model covers exact $0.5 \mathrm{~m}$ ideal tube, whereas the CFD model covers elongated tube with pressure membranes chamber. The velocity distribution along the tube axis follow a sinusoidal pattern, similar to the pressure distribution. Because the pressure and velocity quantities are coupled by the conservation of energy equations the energy transforms form kinetic form to potential pressure form and back.

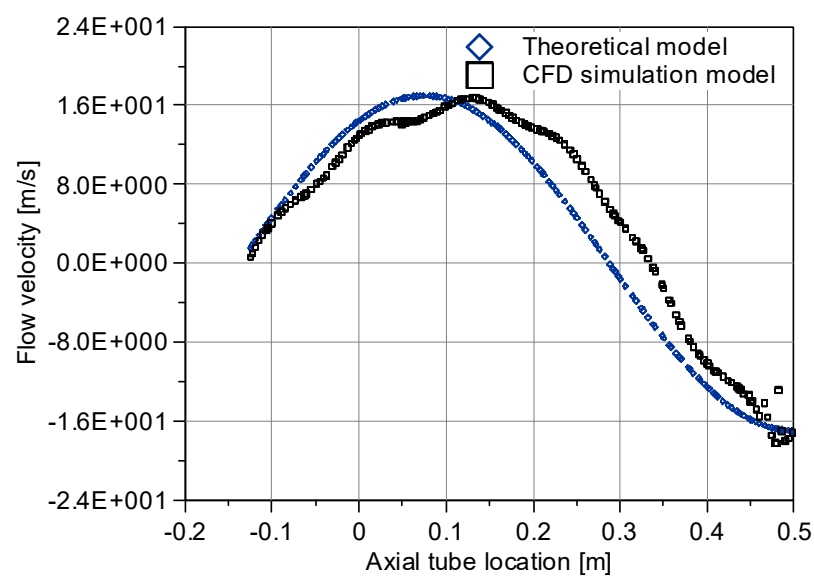

Fig. 7. Flow velocity distribution along the tube axis. The open end is on the right side.

This causes the velocity distribution to be shifted in phase when compared to the pressure distribution.

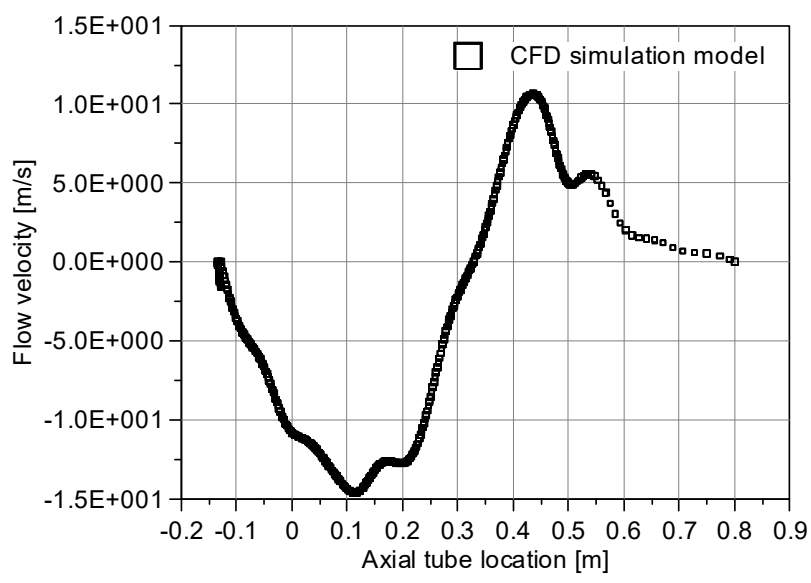

Fig. 8 Flow velocity distribution along the tube axis extended to the open field region. The open end is on the right side at $0.5 \mathrm{~m}$.

The pressure node is velocities antinode and opposite. The presented mathematical model follows this rule with good accuracy. When the wave leaves the tube the pressure and velocity amplitude drop is inversely proportional to the distance (Fig. 8). The outside conditions are hemispherical open field. Some noise is visible along the 
path with higher frequency than the extortion frequency. It is caused by interference and geometry variations [12]. Fig. 9 shows how the velocity changes outside the tube in CFD simulation model. The fit was performed with the equation $\mathrm{Y}=\mathrm{A} / \mathrm{X}+\mathrm{B}$ where $\mathrm{A}=0.294$ and $\mathrm{B}=-0.711$. The residual sum of squares $=0.248936$ and coefficient of determination R-squared $=0.963533$.

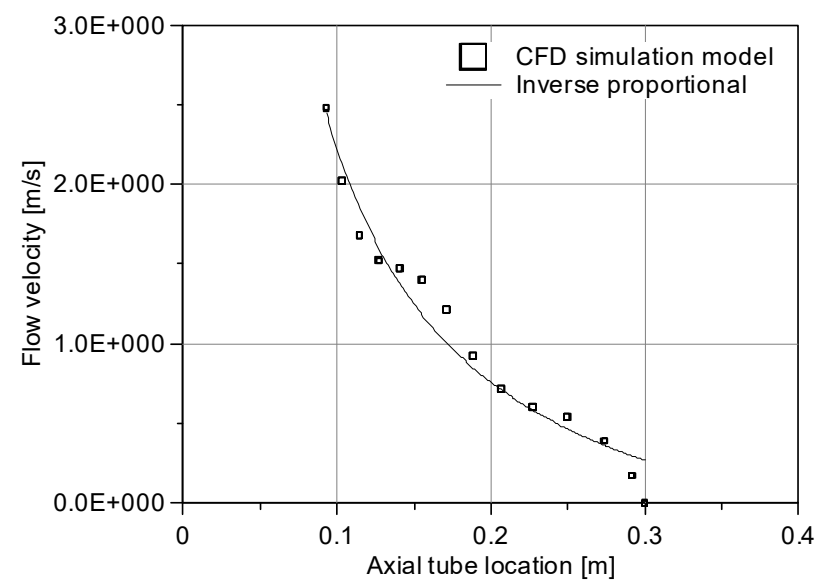

Fig. 9. A flow velocity ploted against distance from the tube exit. Inverse square fit is shown.

Figure 10 presents the CFD simulation pressure field during $400 \mathrm{~Hz}$ membrane extortion operation. On the top of the tube two membranes generate pressure fluctuations. The pressure and velocity are than recorded along the tube's axis.

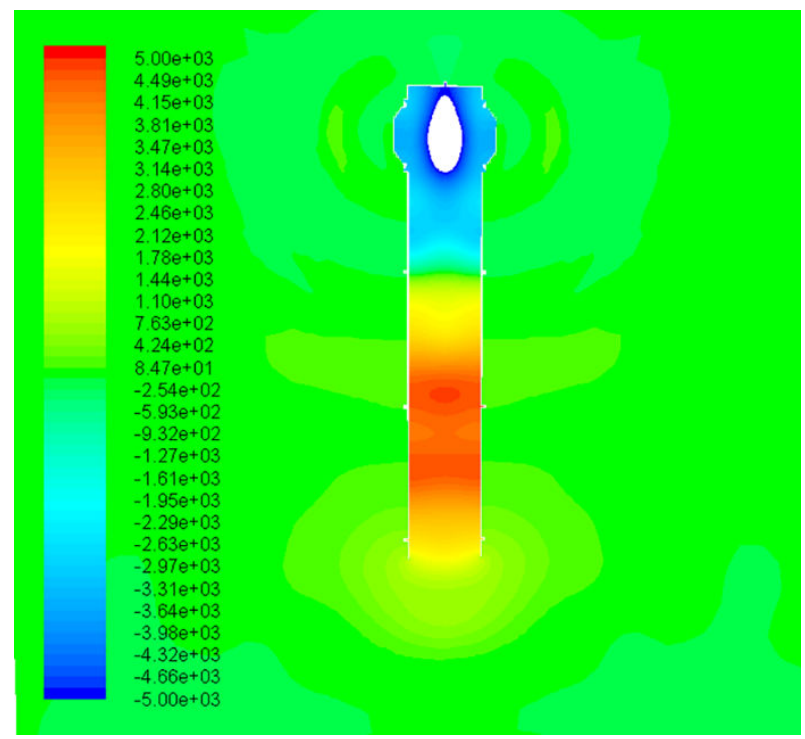

Fig. 10. Relative pressure field for an open end tube CFD simulation.

The research results indicate that the theoretical mathematical model accurately represents the pressurevelocity fluctuations inside the open-end tube. This is possible thanks to transient term in the Bernoulli equation together with the change of density term. The assumptions regarding the acceleration profiles are proven to be correct for analysed case. For increased accuracy the open-field part of the integral 8 can be modified to follow inverse distance equation. Further investigations are required to accurately estimate the $\mathrm{R}$ distance integral limit for different conditions. Although the pressure accumulated through the resonance phenomenon is relatively high for the energy input, it could not be used to generate work for every cycle without losing intensity. Any disruption of the pressure like adding one-way flow valve causes the pressure to drop significantly or act in opposite direction during next cycles. That is because the pressure builds up through many cycles and cannot be used and restored by one cycle.

\section{Conclusions}

The research provides several insights and phenomena descriptions:

- The theoretical mathematical model accurately represents the pressure-velocity fluctuations inside the open-end tube.

- The assumptions regarding the acceleration profiles are proven to be correct for analysed case.

- The linear representation of the open-field pressure decay seems to represent the case accurately, but for increased accuracy the integral 8 can be modified to follow inverse distance equation.

- CFD and theoretical models seem to overlap accurately with slight shift along $\mathrm{x}$ axis.

- The pressure accumulated through the resonance phenomenon is relatively high for the energy input, but it could not be used to generate work for every cycle without losing intensity

\section{References}

1. P. A. Feenstra, D. S. Weaver, and F. L. Eisinger, J. Fluids Struct., 21, 89-101, (2005).

2. R. Hanson and S. Ziada, J. Fluids Struct., 27, 367-382, (2011).

3. A. Mohany and S. Ziada, J. Fluids Struct., 25, 461478, (2009).

4. A. Oengören and S. Ziada, J. Fluids Struct, 6, no. 3, 1992.

5. A. Mohany and S. Ziada, J. Fluids Struct., 27, 11491164, (2011).

6. S. Chen, D. Zhao, H. K. H. Li, T. Y. Ng, and X. Jin, Appl. Therm. Eng., 112, 1070-1082, (2017).

7. D. Zhou, Z. Luo, M. Fang, M. Lu, J. Jiang, H. Chen, and M. He, Appl. Energy, 185, 2245-2250, (2017).

8. J. J. Heys, N. Holyoak, A. M. Calleja, M. Belohlavek, and H. P. Chaliki, Open Biomed. Eng. J., 4, 123-128, (2010).

9. R. C. Stevenson, Z. G. Yang, and V. Jairazbhoy, Appl. Math. Model., 32, 123-140, (2008).

10. MIT course, Unsteady Bernoulli Equation https://ocw.mit.edu, (2003), 11.2017.

11. S. Fontana, D. Moeller, I. Liu, and K. Robins, Acoustic Levitation: A Theoretical Exploration, (2016).

12. D. M. Howard and J. A. . Angus, Acoustics and Psychoacoustics Fourth Edition. (2009). 